\title{
The Radio Continuum Morphology of the Orion Nebula
}

\author{
Marcello Felli \\ Osservatorio di Arcetri, Largo E. Fermi 5, 50125, Florence, Italy
}

Ed Churchwell

University of Wisconsin, 475 N. Charter St., Madison, WI 59706, U.S.A.

Douglas O. S. Wood

Center for Astrophysics, 60 Garden St., Cambridge, Mass 02198, U.S.A.

The Orion Nebula is the bench mark galactic HII region in which major advances have been made in our understanding of the ionization structure, energy balance, plasma dynamics, star formation and interactions of ionized plasmas with dense molecular clouds. Most of these studies require accurate comparison with radio continuum images.

The basic properties of the radio emission from the Orion Nebula, such as the overall shape and flux density, have been accurately determined with single dish observations for a long time. The highest resolution single dish map has been obtained with the 100 m Bonn radiotelescope at $23 \mathrm{GHz}$, with a resolution of 43 " (Wilson and Pauls, 1984). This map corresponds very well with $\mathrm{H} \alpha$ emission. The emission from M42 and M43 are clearly separated and some structure is found in M42, in particular: a bright bar (I-front) close to $\theta^{2}$ Or $i$ and a central core about $3^{\prime}$ in diameter. The total flux density in M42 is $400 \mathrm{Jy}$ and the mean Emission Measure (EM) is $\sim 510^{6} \mathrm{pc} \mathrm{cm}^{-6}$.

Several high resolution interferometric radio maps of the HII region have been published (Martin and Gull, 1976; Righini-Cohen, Simon and Felli, 1981; Garay et al, 1982; Johnston et al, 1983; Garay, Moran and Reid, 1987; Churchwell, Felli, Wood and Massi, 1987; Barvainis and Wootten, 1987; Ohashi et al, 1989). These maps are incomplete because an interferometer acts as a spatial frequency filter and selects only a portion of the spatial frequency spectrum contained in the true source brightness distribution. This effect translates into filtering out the low EM structures as the interferometer baseline increases. Consequently, interferometers with longer baselines are sensitive only to compact, high EM components. This effect is, in many ways, similar to the relationship between sensitivity to low EM and exposure time in optical photographs. In this case, the longer the exposure time, the lower the EM that can be detected, but at the same time the high emission regions are usually saturated.

Another consequence of this effect is that radio maps made with different resolutions will contain different total flux densities. For extended sources like the Orion Nebula, the higher the resolution, the lower the total flux that will be detected.

In this paper we present a comparative study of the radio structure of the nebula over scale sizes from 0.1 " to $45^{\prime}$. The data were obtained with the VLA in its four standard configurations, from the most extended (A) to the most compact (D), at two widely spaced wavelenghts. At $20 \mathrm{~cm}$ we imaged a field of about 30' with resolutions ranging from $45^{\prime \prime}$ to $1^{\prime \prime}$, while at $2 \mathrm{~cm}$ we were able to observe only the inner 3 ' central core with resolutions from 4" to 0.1 ". Here we explore the surface brightness distribution of the nebula from single dish resolutions to the highest presently achievable. 


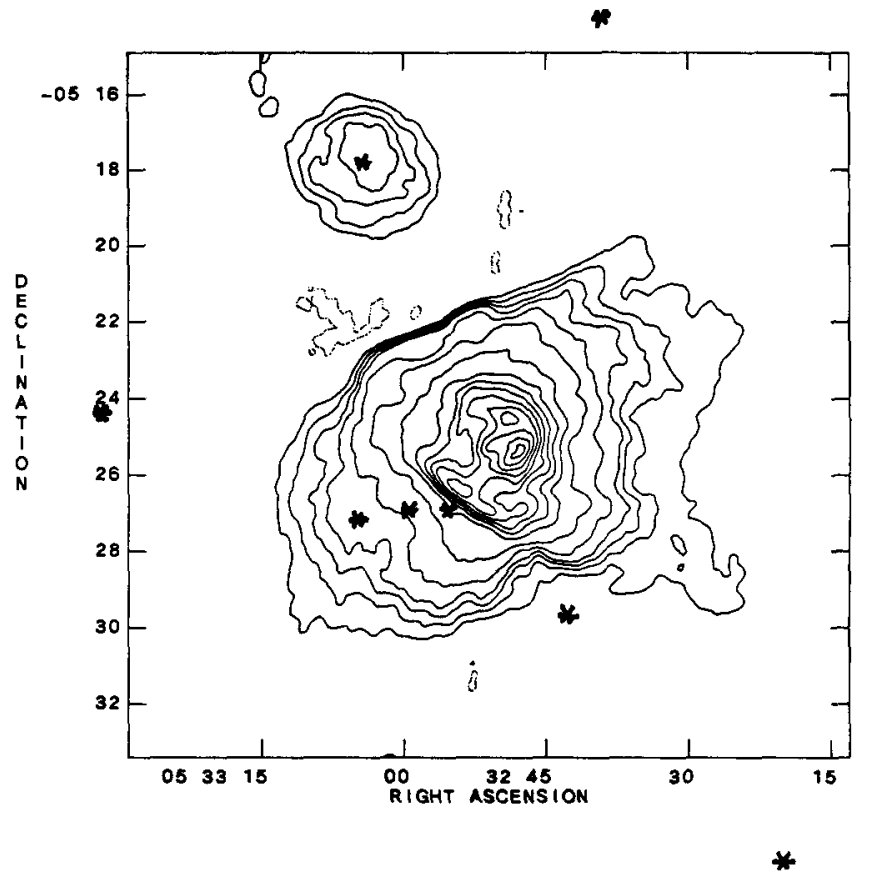

Figure 1a

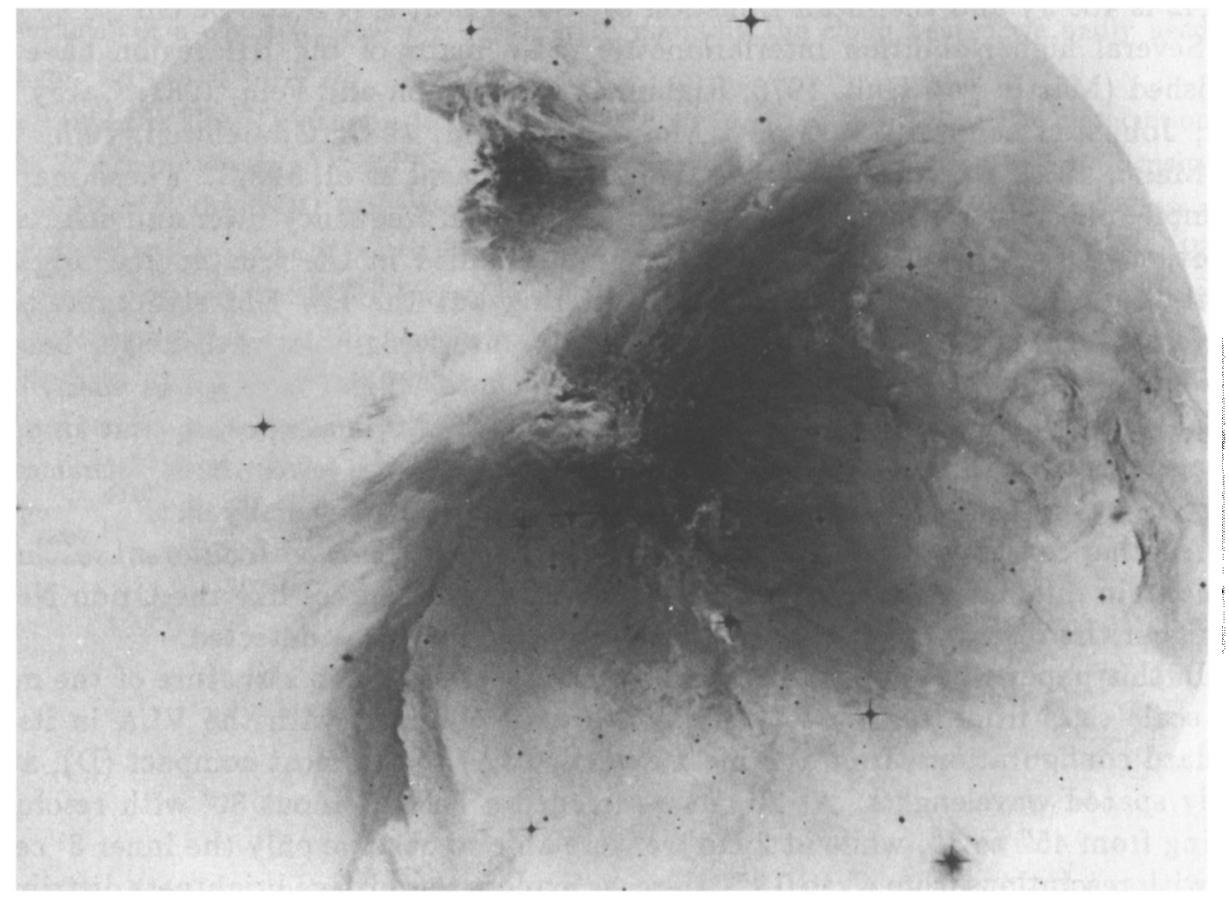

Figure 1b 
In principle one would like to have all the brightness distribution information combined into a single image, at each frequency. In practice this turns out to be almost impossible. The basic problem is that the EM dynamic range from the brightest compact component to the faintest diffuse emission is too large to be recorded with all strucures easily visible on any single image. This is true for optical photographs, CCD images, and radio images. For sources like the Orion Nebula, where a very large range of EM are present, the combination of two or more adjacent VLA configurations is always dominated by the lower resolution configuration. Although we have attempted to do this in a few instances, here we will present only the maps obtained from single VLA configurations. Also, due to the limited space and to the fact that some maps are still being reduced, we will present only a sample of the information that can be extracted from these data.

At the lowest resolution the most obvious features are the roundish structureless shape of M43, the bright bar and central bright core in M42. Figure 1a shows our 20 cm C-configuration map (resolution 20"). The $*$ s indicate the positions of reference stars to be used in the comparison with the $\mathrm{H} \alpha$ photograph of figure $1 \mathrm{~b}$ (from ESO). Both images have the same angular scale. In general the two images are in very good agreement, implying that, on a large scale, foreground dust absorption plays a minor role. This is consistent with the widely accepted idea that the Trapezium stars are eroding a molecular cloud in the backgroud and that the ionized gas is flowing toward the observer. We also call attention to the very sharp gradient along the NE boundary of M42 which probably indicates dense neutral gas at this ionization-bounded front, although part of this effect could also be due to the negative depression produced by the interferometer response to M42 and M43. The mean EM in the map is $1.310^{7} \mathrm{pc}$ $\mathrm{cm}^{-6}$.

We will now concentrate on M42. At a resolution of 7 " the diffuse extended structure begins to fade away and only the bright bar and the compact core stand out clearly. Figure $2 \mathrm{a}$ shows the $20 \mathrm{~cm}$ B-configuration image and figure $2 \mathrm{~b}$ shows the $\mathrm{H} \alpha$ short exposure photograph of G. Munch (Munch and Wilson, 1962), reproduced with the same scale as the radio image. The structure of the ionization front and the small scale components in the central core compare very well in both images. Two "jets" of emission perpendicular to the bright bar are evident in the radio map that are not clearly visible on the optical photo. In the dark bay area, NE of the Trapezium stars, there is a deficiency of ionized gas. The optical dark bay is apparently not caused by foreground extinction but by a deficiency of high EM ionized gas.

For higher resolutions, we now shift to the $2 \mathrm{~cm}$ maps. Due to the limited field of view ( $\left.3^{\prime}\right)$ we can now see only the central core. The bright bar is beyond the HPW of the primary beam. Figure 3 shows the $2 \mathrm{~cm} \mathrm{D-configuration} \mathrm{map} \mathrm{(resolution} \mathrm{4"),} \mathrm{overlayed}$ on Munch's $\mathrm{H} \alpha$ photograph. The agreement between optical and radio structures is impressive. Only some areas in the outer NE part of the core may be affected by foreground obscuration. For the rest of the structures there is almost a one to one correspondence. The Trapezium stars are located slightly to the south of the center of the backward C-shaped ionized core. The core is rather sharply bounded to the west but is more irregularly bounded to the east. $\Theta^{1}$ OriC, the earliest spectral type star of the cluster, is located in a region of lower emission in the core, suggesting that either radiation pressure and/or a stellar wind might have evacuated a region around the star 

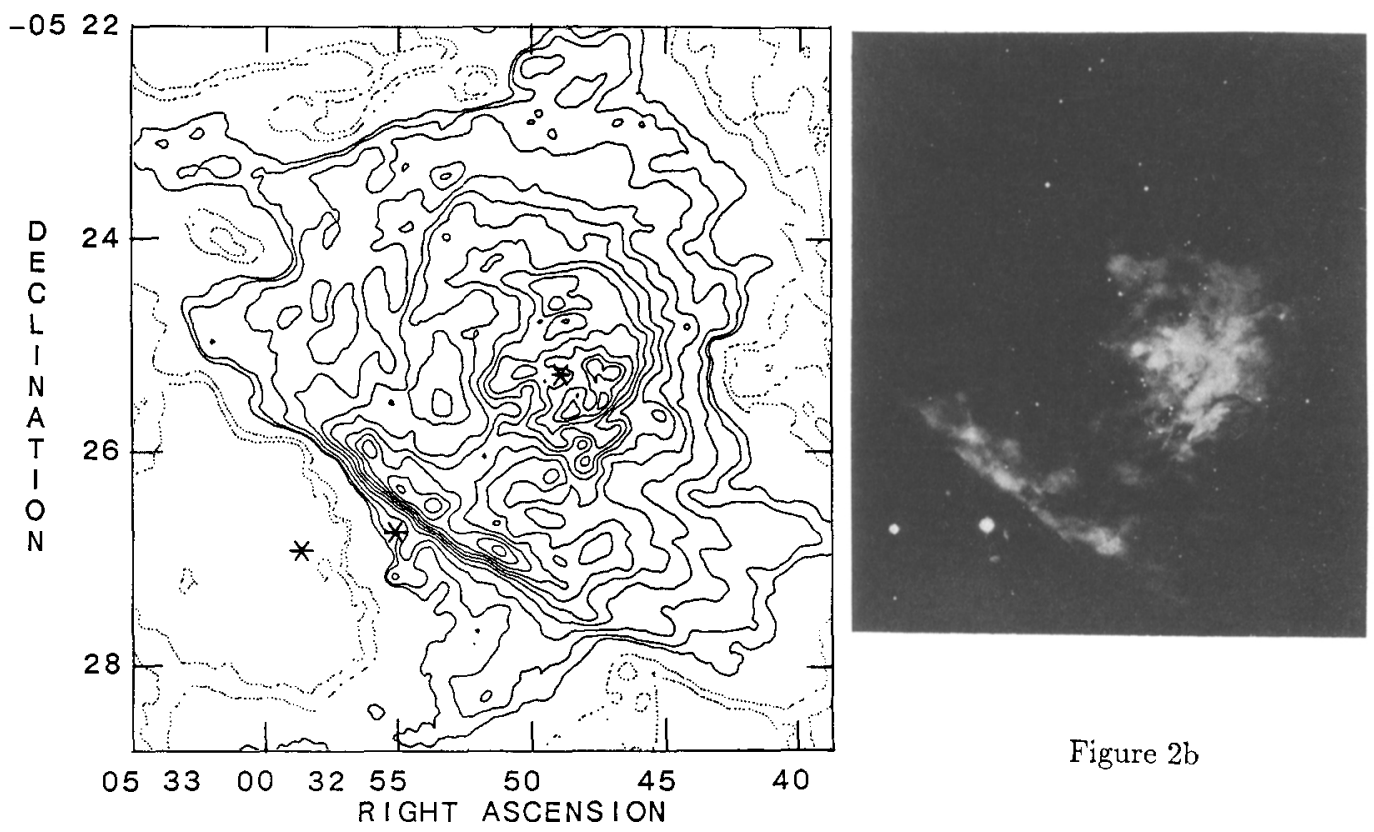

Figure 2a

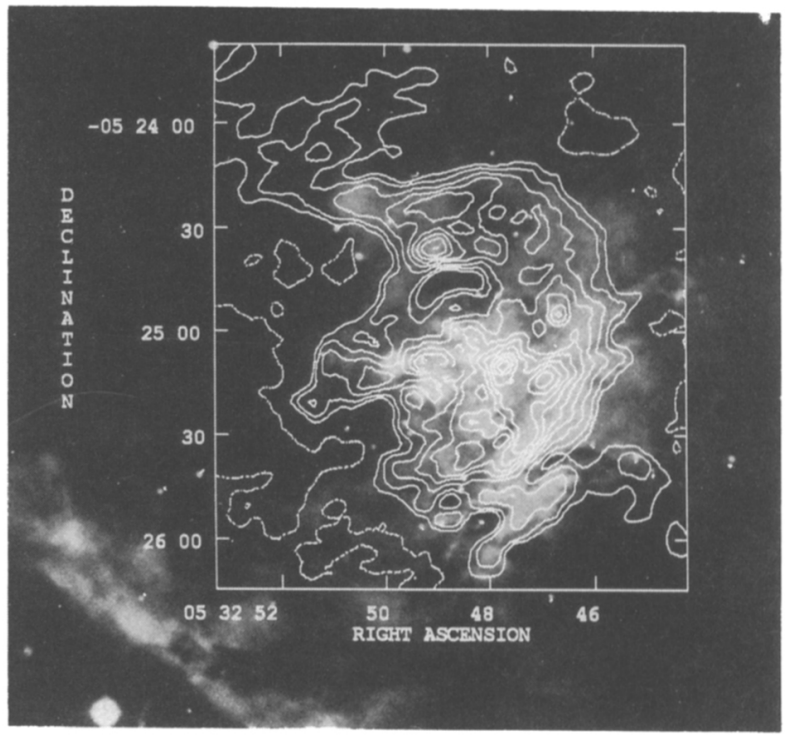

Figure 3

Figure $2 b$ 
(Barvainis and Wootten, 1987). The structured appearance of the core on this scale size (approximately $10^{-2} \mathrm{pc}$ ) must originate from neutral blobs which are embedded in the ionized nebula and have thin ionized envelopes (i.e PIGs). If they were fully ionized density enhancements, their lifetimes against expansion would be too short to understand the large number observed.

Of the many comparisons that can be made between figure 3 and other types of emission in the Orion core, we show in figure 4 a skematic comparison of the continuum emission with the ${ }^{12} C^{18} O$ (1-0) map of Wilson et al (1986) (which shows the morphology of the high density, $\sim 10^{6} \mathrm{~cm}^{-3}$, molecular gas) and with the $20 \mu \mathrm{m}$ map of the BN-KL region ( Downes et al, 1981). It is obvious that the ionized core is bounded to the $\mathrm{W}$ by a dense molecular cloud, which probably determines the structure of the ionized core. The BN-KL region is located at the interface between the ionized and molecular gas. No radio continuum emission from BN or IRc2 is apparent at this resolution; this map is still dominated by diffuse emission. A similar result holds for the bright bar, in which the ionized gas is bounded to the south by a CO molecular cloud (Ohashi et al, 1989).

At even higher resolutions (1"), a new and unexpected aspect of the surface brightness distribution begins to appear. In figure 5 , the $2 \mathrm{~cm}$ C-configuration is shown overlayed on Munch's photograph. While the radio emission of the bright extended blobs predominant in figure 3 , they begin to fade away due to increased resolution, and a new class of small or unresolved components begins to stand out. They were not apparent in the lower resolution maps and they do not necessarely coincide with the peaks of those maps. A cluster of these components is clearly evident in the Trapezium region, one of which coincides with $\theta^{1}$ OriA, while others are located in regions not connected with bright extended nebulosities. At this resolution, both BN and IRc2 are detected as well as a host of other compact sources. These sources represent a wide variety of objects and emission mechanisms, most of which are not yet understood. Several have been discussed in some detail by Laques and Vidal (1987); Garay, Moran, and Reid $(1985,1987)$; and Churchwell et al (1987). These objects have been referred to as the "Orion Zoo" by Garay (1987) and they will be discussed further by him later in this meeting. In the spirit of comparing the structure at different resolutions, we simply point out that at $2 \mathrm{~cm}$ in the A-configuration, all the diffuse emission is gone and only the compact sources with $\mathrm{EM}>10^{8} \mathrm{pc} \mathrm{cm^{-6 }}$ and angular diameters $<0.5^{\prime \prime}$ are left. The total flux density contained in this map is about $180 \mathrm{mJy}$, less that three orders of magnitude smaller than the total flux density of the nebula.

The coincidence between the radio source and the star $\theta^{1}$ OriA can be established with an accuracy of $\sim 0.2^{\prime \prime}$ (this limit is imposed by the optical position of the star). However from VLA data alone the interpretation of the radio emission was unclear. While thermal emission from an ionized wind or an ultracompact HII region seemed the most obvious possibilities, these conflicted with the brightness temperature derived from the VLA size $\left(T_{b}>54,000 \mathrm{~K}\right)$ and the fact that, during a period of $\sim 5$ years, the radio emission varied from a minimum of $3 \mathrm{mJy}$ to a maximum of $74 \mathrm{mJy}$ (see figure 7). To obtain a better estimate of the angular diameter, and therefore of the brightness temperature, we have used the European VLBI Network at $6 \mathrm{~cm}$ to observe this source. The visibility curve is consistent with an angular diameter $<4$ mas (i.e. $1.8 \mathrm{AU}$ ) and a flux density of $13 \mathrm{mJy}$ (equal to that measured simultaneously with the VLA). These data imply a brightness temperature $>4 \times 10^{7} \mathrm{~K}$ on 28 Sept. 1987, which unambiguously 


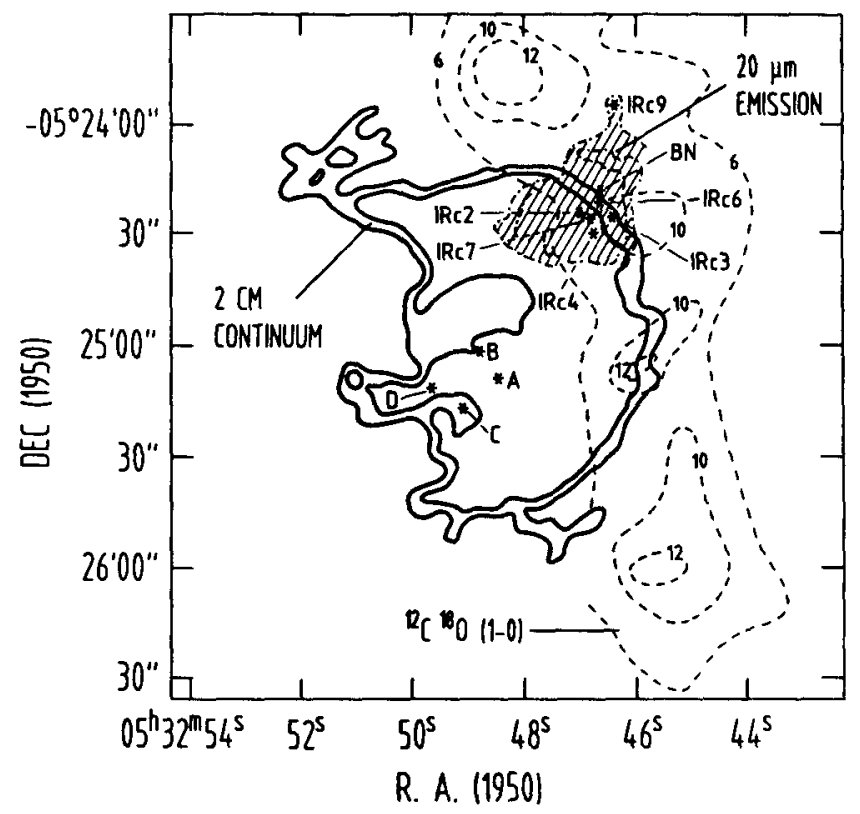

Figure 4

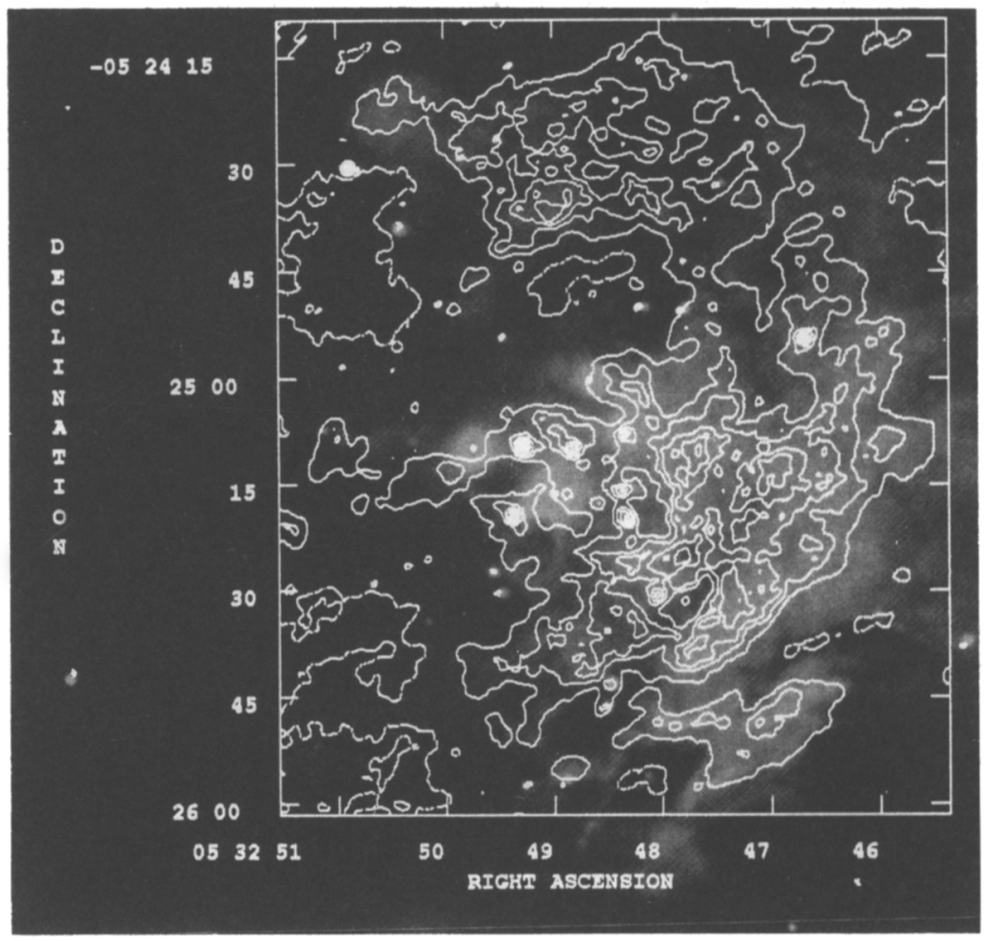

Figure 5 


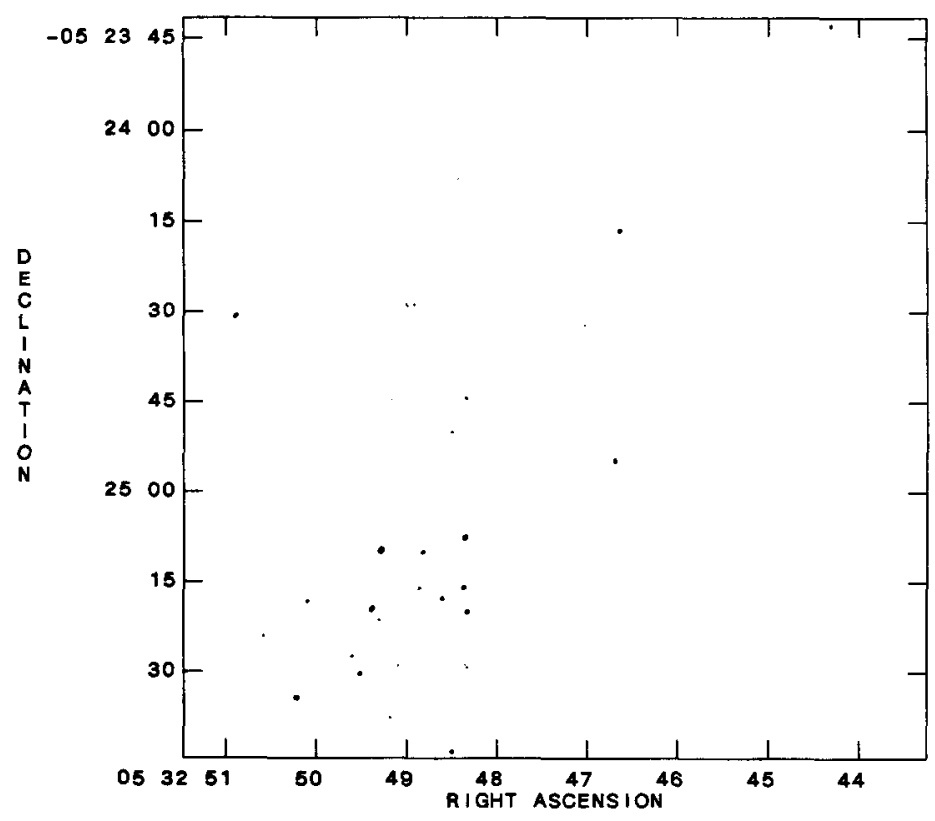

Figure 6

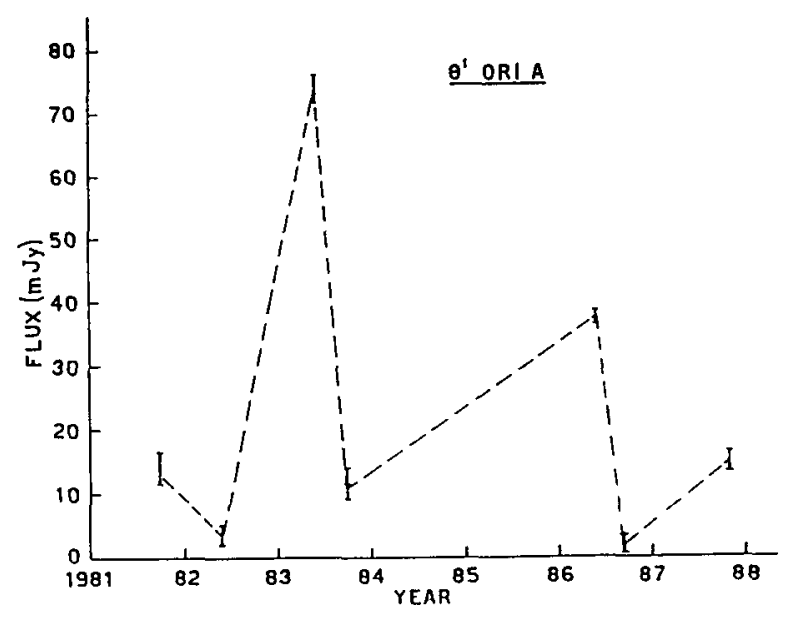

Figure 7

$<1.8 \mathrm{AU}$ ) and a flux density of $13 \mathrm{mJy}$ (equal to that measured simultaneously with the VLA). These data imply a brightness temperature $>410^{7} \mathrm{~K}$ on 28 Sept. 1987, which unambiguously proves that the emissiom mechanism must be non-thermal (Felli, Massi and Churchwell, 1989). $\Theta^{1}$ OriA is a binary system composed of a B3III-V star and a possible $\mathrm{T}$ Tauri companion whose mean separation is $0.71 \mathrm{AU}$. While it is too early to establish what the non-thermal mechanism is in the star, one possibility may be syncrotron emission from relativistic electrons in large magnetic loops within the binary system energized by flare events on the $T$ Tauri star. 


\section{References}

Barvainis, R., Wootten, A., 1987, A. J., 93, 168

Churchwell, E., Felli, M., Wood, D.O.S., Massi, M., 1987, Ap. J., 321, 516

Downes, D., Genzel, R., Becklin, E.E., Wynn-Williams, C.G., 1981, Ap. J., 244, 869

Dyson, J.E., 1968, Ap. Space Sci.,1, 388

Felli, M., Massi, M., Churchwell, E., 1989, Ast. Ap. , in press

Garay, G., Reid, M.J., Genzel, R., Ho, P.T.P., 1982, Symposium on the Orion Nebula to Honor Henry Draper, Glassgold, A.E., Huggins, P.J., Schucking, E.L., Eds., The New York Academy of Sciences, 204.

Garay, G., Moran, J.M., Reid, M.J., 1985, in Radio Stars, ed R.M. Hjellming and D.M. Gibson, Dordrecht, Reidel, p131

Garay, G., Moran, J.M., Reid, M.J., 1987, Ap. J., 314, 535

Garay, G., 1987, Rev. Mex. Astron. Astrophys., 14, 489

Johnston,K.J., Palmer, P., Wilson, T.L., Bieging, J.H., 1983, Ap. J., 271, L89

Laques, P., Vidal, J. L. 1978, Ast. Ap., 73, 97

Martin, A.H.M., Gull, S.F., 1976, Mon. Not. R. A. S., 175, 235

Moran, J.M., Garay, G., Reid, M.J., Genzel, R., Wright, M.C.H., Plambeck, R.L., 1983, Ap.J., 271, L31

Munch, G., Wilson, O.C., 1962, Z. Astrophys., 56, 127

Ohashi, N., Mizuno, A., Tatematsu, K., Sugitani, K., Kasugu, T., Fukui, Y., 1989, Ap. $\mathrm{J}$., in press

Righini-Cohen, G., Simon, M., Felli, M., 1981, Sky and Telescope, 62, 225

Wilson, T.L., Pauls, T., 1984, Ast. Ap., 138, 225

Wilson, T.L., Serabyn, E., Henkel, C., Walmsley, C.M., 1986, Ast. Ap. 158, L1 